\title{
Expression quantitative trait loci infer the regulation of isoflavone accumulation in soybean (Glycine max L. Merr.) seed
}

\author{
Yan Wang ${ }^{\dagger}$, Yingpeng Han ${ }^{\dagger}$, Weili Teng, Xue Zhao, Yongguang Li, Lin Wu, Dongmei Li and Wenbin Li
}

\begin{abstract}
Background: Mapping expression quantitative trait loci (eQTL) of targeted genes represents a powerful and widely adopted approach to identify putative regulatory variants. Linking regulation differences to specific genes might assist in the identification of networks and interactions. The objective of this study is to identify eQTL underlying expression of four gene families encoding isoflavone synthetic enzymes involved in the phenylpropanoid pathway, which are phenylalanine ammonia-lyase (PAL; EC 4.3.1.5), chalcone synthase (CHS; EC 2.3.1.74), 2-hydroxyisoflavanone synthase (IFS; EC1.14.13.136) and flavanone 3-hydroxylase ( $F 3 \mathrm{H} ; \mathrm{EC} 1.14 .11 .9$ ). A population of 130 recombinant inbred lines $\left(\mathrm{F}_{5: 11}\right)$, derived from a cross between soybean cultivar 'Zhongdou 27' (high isoflavone) and 'Jiunong 20' (low isoflavone), and a total of 194 simple sequence repeat (SSR) markers were used in this study. Overlapped loci of eQTLs and phenotypic QTLs (pQTLs) were analyzed to identify the potential candidate genes underlying the accumulation of isoflavone in soybean seed.
\end{abstract}

Results: Thirty three eQTLs (thirteen cis-eQTLs and twenty trans-eQTLs) underlying the transcript abundance of the four gene families were identified on fifteen chromosomes. The eQTLs between Satt278-Sat_134, Sat_134-Sct_010 and Satt149-Sat_234 underlie the expression of both IFS and CHS genes. Five eQTL intervals were overlapped with pQTLS. A total of eleven candidate genes within the overlapped eQTL and PQTL were identified.

Conclusions: These results will be useful for the development of marker-assisted selection to breed soybean cultivars with high or low isoflavone contents and for map-based cloning of new isoflavone related genes.

Keywords: Soybean, eQTL, Isoflavone, pQTL, Candidate genes

\section{Background}

Soy food has been taken as a functional food because it contains many health beneficial molecules such as isoflavones [1]. Studies on human nutrition have shown that soybean isoflavones play an important role in preventing a number of chronic diseases [2,3]. Equally, isoflavones are critical factors in defending soybean crops against pests $[4,5]$, in promoting nodulation by rhizobia [6], and in changing or adjusting the microorganisms around plant roots [7]. The major bioactive components of soybean isoflavones in human nutrition are daidzein (DZ), genistein (GT) and glycitein (GC). Isoflavone contents in soybean

\footnotetext{
* Correspondence: wenbinli@neau.edu.cn

'Equal contributors

Key Laboratory of Soybean Biology in Chinese Ministry of Education (key Laboratory of Soybean Biology and Breeding/Genetics of Chinese Agriculture Ministry), Northeast Agricultural University, Harbin 150030, China
}

seed are inherited as complex quantitative traits [8-11]. Since soy seed isoflavones are regulated by multiple genetic factors, their concentrations in seed are highly variable [1,12-14]. Over fifty QTLs underlying individual and/ or total soybean isoflavone content have been reported [8,15-23]. However, only 12 of these QTLs were in genomic regions encoding isoflavone synthesis enzymes.

A group of enzymes in the phenylpropanoid pathway lead to the biosynthesis of DZ, GT and GC [11]. Phenylalanine ammonia lyase (PAL; EC 4.3.1.5), chalcone synthase (CHS; EC 2.3.1.74) and flavanone 3-hydroxylase (F3H; EC 1.14.11.9) [24] are the first three enzymes that convert the amino acid phenylalanine into p-CoumaroylCoA in this pathway [11]. In the isoflavonoid biosynthetic pathway [25], the co-catalytic action of $\mathrm{CHS}[26,27]$ with chalcone reductase (CHR; EC 2.3.1.170) [28] produces isoliquiritigenin and naringenin chalcone, which are isomers 
of the central isoflavanone intermediates naringenin and liquiritigenin, respectively. Isoliquiritigenin and naringenin chalcone are respectively converted into liquiritigenin and naringenin by chalcone isomerase (CHI; EC 5.5.1.6) [29]. These two products are the precursors of DZ and GT, which are formed after the catalysis of the precursors by the key enzyme 2-hydroxyisoflavanone synthase (IFS; EC 1.14.13.136) [30,31]. The enzyme F3H, that competes with IFS in utilizing naringenin, catalyzes the conversion of flavanones to dihydroflavonols, which are intermediates in the biosynthesis of flavonols, anthocyanidins, catechins and proanthocyanidins [32,33]. For the synthesis of GC, isoliquiritigenin is likely a precursor to form GC after several biochemical steps, which are not entirely known yet [34]. However, seed isoflavone concentrations in soybean can be regulated by metabolic engineering of the complex phenylpropanoid biosynthetic pathways [35].

Regulating transcript abundance is an effective approach to improve phenotypes [36]. The integrated analysis of genotype and transcript abundance data for association with complex traits can be used to identify novel genetic pathways involved in complex traits. 'Expression QTL' (eQTL), first defined by Jansen and Nap [37], could identify the genetic determinants of transcript abundances and is widely used for investigating gene regulation pathways. This approach treats transcript abundance of individual genes as quantitative traits in a segregating population. The eQTL map information enables genetic regulatory networks to be modeled that can provide a better understanding of the underlying phenotypic variation. It has been successfully applied in humans [38-40], plants [41-44], yeasts [45,46], worms [47], flies [48], mice [49,50], pigs [51] and rats [52] populations. These studies showed that transcript abundance was highly heritable and could be linked to either a local locus (cis-eQTL) or a distant locus (transeQTL). Cis-eQTL is mapped to the same genomic location like an expressed gene (within $5 \mathrm{Mb}$ ), and transeQTL is mapped to a different genomic location from an expressed gene ( $>5 \mathrm{Mb}$ or on different chromosomes) $[40,53]$. In general, cis-eQTL tends to produce stronger statistical associations than does by trans-eQTL [54]. This phenomenon is regarded as evidence of greater biological plausibility for the existence of true functional cis-eQTL [55]. Trans-eQTL could occur individually at a single genomic locus or could occur collectively as part of eQTL trans-bands [55]. This genomics approach has been employed to identify eQTL related genes in soybean [36,56-58]. To date, no information concerning eQTLs underlying soybean isoflavone synthetic enzyme genes is available.

It has been proved that many enzymes in the phenylpropanoid pathway underlie QTLs that determine the accumulation of isoflavone contents in soybean seeds
[11]. Meanwhile, the modification of enzyme encoded genes that are involved in phenylpropanoid pathway could promote the biosynthesis of isoflavone [31,35]. In this study, PAL, CHS, IFS and F3H in the phenylpropanoid pathway were selected as the target genes (TGs) to analyze isoflavone-relative eQTL. Potential candidate genes underlying the accumulation of isoflavone contents in soybean seed were also evaluated. In addition, overlapped loci both for eQTL and phenotypic QTL (pQTL) were identified.

\section{Results}

Total and individual isoflavone contents, target gene transcript abundance and correlation analysis

Transcript abundances of target genes (TGs) between parents from R3 to R8 developmental stages were compared. Total and individual isoflavone contents and transcript abundances of TGs at R6 stage of soybean development were measured in the $F_{5: 11}$ population. The results showed that significant differences among the transcript abundances of TGs between the two parents existed at the R6 stage. The phenotypic variation of individual and total isoflavones showed a continuous distribution (Table 1).

GT showed a high positive correlation coefficient with DZ ( $\mathrm{r}=0.762, \mathrm{P}<0.01$; Table 2). The transcript abundance of $P A L$ was positively correlated with both GT and TI, but exhibited no significant correlation with DZ and GC. The transcript abundance of $C H S$ was positive correlated with DZ, GT and TI, but negatively associated with GC amount. The transcript abundance of IFS displayed a positive correlation with $\mathrm{DZ}$, but showed no correlation with other isoflavone components. The transcript abundance of $F 3 H$ showed significantly negative correlation with individual and total isoflavone contents.

\section{Identification of genomic region for target genes}

Through BLAST searches (http://www.phytozome.net/ soybean), the $P A L$ has six homologous regions $(\mathrm{E} \leq 0)$, which are located on Gm10 (LG O, PAL1/ PAL2), Gm13 (LG F, PAL1), Gm03 (LG N, PAL1), Gm19 (LG L, PAL1), Gm20 (LG I) and Gm02 (LG D1b, PAL1). Homologous regions encoding $\mathrm{CHS}$ (E-value $\leq 1.0 \mathrm{E}-05)$ are located on Gm11 (LG B1, CHS8), Gm01 (LG D1a, CHS6/CHS7), Gm08 (LG A2, CHS1/CHS2/CHS3/CHS4/CHS5/CHS9), Gm05 (LG A1, CHS2), Gm02 (LG D1b), Gm09 (LG K, CHS6), Gm19 (LG L) and Gm13 (LG F). Genes that encode $F 3 H$ are located on Gm02 (LG D1b, F3H1/F3H2), Gm16 (LG J), Gm01 (LG D1a), Gm11 (LG B1), Gm18 (LG G) and Gm19 (LG L). Genes encoding IFS are located on Gm07 (LG M IFS1), Gm13 (LG F, IFS2), Gm10 (LG O), Gm03 (LG N), Gm12 (LG H), Gm19 (LG L), Gm17 (LG D2) and Gm11 (LG B1). Genes encoding IFS have the function of P450 cytochromes [27] and might have additional functional homologs. 
Table 1 Total and individual isoflavone content of the RIL populations and parents

\begin{tabular}{|c|c|c|c|c|c|c|c|c|}
\hline Traits $^{a}$ & Mean $^{\mathbf{b}}$ & $S D^{b}$ & $\operatorname{Min}^{b}$ & $\operatorname{Max}^{b}$ & Zhongdou $27^{c}$ & Jiunong $20^{c}$ & Skewness & Kurtosis \\
\hline DZ & 9.61 & 3.04 & 4.36 & 15.88 & $8.92 \pm 2.97$ & $4.79 \pm 1.12$ & -0.040 & -0.765 \\
\hline GC & 0.41 & 0.32 & 0.29 & 2.64 & $0.36 \pm 0.16$ & $0.42 \pm 0.23$ & 0.138 & 0.825 \\
\hline GT & 4.38 & 2.55 & 0.77 & 9.51 & $4.22 \pm 2.75$ & $2.81 \pm 1.01$ & 0.480 & -0.860 \\
\hline $\mathrm{Tl}$ & 14.40 & 5.21 & 5.70 & 25.11 & $13.50 \pm 5.21$ & $6.81 \pm 2.27$ & 0.187 & -1.061 \\
\hline$P A L$ expression $(\triangle \triangle C T)$ & 3.926 & 7.388 & 0.009 & 37.570 & 0.252 & 0 & 0.590 & 0.846 \\
\hline CHS expression $(\triangle \triangle C T)$ & 0.013 & 0.013 & 0.0002 & 0.051 & 0.328 & 0 & 1.203 & 0.616 \\
\hline IFS expression $(\Delta \Delta C T)$ & 0.896 & 1.334 & 0.002 & 5.199 & 0.707 & 0 & 0.954 & 1.700 \\
\hline$F 3 H$ expression $(\triangle \triangle C T)$ & 4.798 & 3.481 & 0.013 & 10.550 & 10.550 & -16.047 & 0.156 & -1.340 \\
\hline
\end{tabular}

DZ, Daidzein; GC, Glycitein; GT, Genistein; TI, Total isoflavone content.

${ }^{\mathrm{b}} \mu \mathrm{g} / 100 \mathrm{~g}(\mathrm{DZ}, \mathrm{GC}, \mathrm{GT}, \mathrm{TI})$.

${ }^{c}$ Mean \pm SD.

\section{eQTL analysis for four TGs}

The linkage map that included 194 SSR markers (accepted by Molecular Biology Reports) and covered 2,312 cM with mean distance of about $12 \mathrm{cM}$ between markers was used to identify eQTLs associated with the expression of the four TGs. Thirty-three eQTLs that appeared to underlie transcript abundance of the four TGs are detected and located on fifteen LGs (Table 3, Figure 1). Regarding to the locational relationships between the eQTL and the genes, thirteen of the eQTLs were cis-acting (within $5 \mathrm{Mb}$ upstream or downstream of the genes) and twenty of the eQTLs were trans-acting (more than $5 \mathrm{Mb}$ away or on different chromosomes) [40,53].

Among the identified eQTLs (Table 3), qPALB2_1 and qPALD2_1 were associated with PAL transcript abundance, and could explain $8.11 \%$ and $6.67 \%$ of the phenotypic variation, respectively. Eight eQTLs, underlying CHS transcript abundance, were located on six LGs, and could explain $2.07-15.65 \%$ of the phenotypic variation. qCHSDla_1 (Satt436-Sat_345, Gm01) was detected with a higher LOD score (8.64) in the regions where ciselements and $C H S$ family genes were located.

Two eQTLs (qCHSDlb_1, qCHSDlb_2), located in the interval of Satt459 and Satt546, could explain 2.13\% and
3.90\% of phenotypic variance and overlap with qGCD1b_1. qCHSF_1 (Satt149-Sat_234), associated with CHS and IFS transcript abundance, were overlapped with the marker interval of qGTF_2, and could explain 3.57\% of phenotypic variance. qCHSL_1 (Satt278-Sat_134) and qCHSL_2 (Sat_134-Sct_010) were associated with the same SSR marker (Sat_134), and contributed $16.12 \%$ and $17.97 \%$ of the variation of IFS transcript abundance.

Twelve eQTLs were associated with IFS expression. Of them, qIFSD2_1 (Satt186-Satt226) explained 16.67\% of the phenotypic variation. qIFSF_1 (Satt423-Satt569, $\mathrm{R}^{2}=$ 15.84\%) shared the same SSR marker Satt569 with other three QTLs (qDZF_2, qGTF_1, qTIF_2). qIFSN shared the same SSR marker (Satt530) with qGCN_1 (Table 3, Figure 1).

Eleven eQTLs were associated with $F 3 H$ expression (Table 3, Figure 1). Of them, four eQTLs were located on Gm02 (LG Dlb), and explained $5.54-14.32 \%$ of the phenotypic variation. qF3HDlb_2 (Sat_135-Sat_096) had higher LOD score and explained $14.32 \%$ of the phenotypic variation. qF3HE_1 $\left(\mathrm{R}^{2}=4.85 \%\right)$ had the same interval (Sat_112-Sat_380) with qGCE_1, qGTE_1 and qTIE_1, meanwhile, qF3HF_1 and qDZF_1 shared the same marker interval (Sat_262-Sat_103) (Figure 1).

Table 2 Correlations among individual and total isoflavone contents, as well as the transcript abundances of the four TGs in the RIL populations

\begin{tabular}{|c|c|c|c|c|c|c|c|}
\hline Traits & $\mathrm{DZ}$ & GC & GT & $\mathrm{TI}$ & $P A L$ expression & CHS expression & IFS expression \\
\hline GC & $0.249^{*}$ & & & & & & \\
\hline GT & $0.762^{* *}$ & $0.294^{*}$ & & & & & \\
\hline $\mathrm{Tl}$ & $0.943^{* *}$ & $0.363^{*}$ & $0.928^{* *}$ & & & & \\
\hline PAL expression & -0.094 & 0.092 & $0.269^{*}$ & $0.304^{*}$ & & & \\
\hline CHS expression & $0.223^{*}$ & $-0.191^{*}$ & $0.201^{*}$ & $0.230^{*}$ & 0.063 & & \\
\hline IFS expression & $0.327^{*}$ & -0.032 & 0.169 & 0.140 & -0.022 & 0.022 & \\
\hline F3H expression & $-0.248^{*}$ & $-0.248^{*}$ & $-0.276^{*}$ & $-0.273^{*}$ & 0.105 & 0.108 & -0.001 \\
\hline
\end{tabular}

$\mathrm{P}$ values were as follows: ${ }^{*} \mathrm{P}<0.05,{ }^{* *} \mathrm{P}<0.01$. 
Table 3 The eQTLs for target genes of PAL, CHS, IFS and F3H

\begin{tabular}{|c|c|c|c|c|c|c|c|c|}
\hline Traits & $\mathrm{eQTL}^{\mathrm{a}}$ & $\mathrm{Gm}(\mathrm{LG})$ & Marker & Marker interval & Position $^{\text {b }}$ & Environment & LOD score & $R^{2}(\%)^{c}$ \\
\hline \multirow[t]{2}{*}{$P A L$} & ${ }^{\mathrm{d}}$ qPALB2_1 & 14(B2) & Satt560 & Satt560 Satt556 & 0.01 & 2011Harbin & 3.39 & 8.11 \\
\hline & daPALD2_1 & $17(\mathrm{D} 2)$ & Sat_209 & Sat_209 Sat_022 & 15.90 & 2011Harbin & 4.24 & 6.67 \\
\hline \multirow[t]{8}{*}{$\mathrm{CHS}$} & qCHSA1_1 & 05(A1) & Satt 236 & Satt 236-D26A & 0.01 & 2011Harbin & 5.48 & 4.21 \\
\hline & qCHSDla_1 & $01(\mathrm{Dla})$ & Satt436 & Satt436-Sat_345 & 0.01 & 2011Harbin & 8.64 & 2.71 \\
\hline & qCHSDIb_1 & 02(Dlb) & Satt546 & Satt546-Satt459 & 214.80 & 2011Harbin & 2.55 & 2.13 \\
\hline & qCHSDlb_2 & 02(Dlb) & Satt546 & Satt546-Satt459 & 211.22 & 2011Harbin & 2.18 & 3.90 \\
\hline & dqCHSD2_1 & $17(\mathrm{D} 2)$ & Satt528 & Satt528-Satt256 & 10.74 & 2011Harbin & 2.73 & 2.07 \\
\hline & qCHSF_1 & $13(\mathrm{~F})$ & Sat_234 & Sat_234-Satt149 & 46.17 & 2011Harbin & 2.72 & 3.57 \\
\hline & qCHSL_1 & $19(L)$ & Satt278 & Satt278-Sat_134 & 14.00 & 2011Harbin & 2.40 & 15.65 \\
\hline & qCHSL_2 & $19(L)$ & Sat_134 & Sat_134-Sct_010 & 24.51 & 2011 Harbin & 2.09 & 9.98 \\
\hline \multirow[t]{12}{*}{ IFS } & ${ }^{d} \mathrm{qIFSA} 2$ 1 & $08(A 2)$ & Sat_129 & Sat_129-Sat_181 & 55.45 & 2011Harbin & 7.46 & 17.67 \\
\hline & dqIFSC1_1 & $04(C 1)$ & Sat_042 & Sat_042-Satt524 & 6.67 & 2011Harbin & 5.63 & 22.8 \\
\hline & dqIFSD2_1 & $17(\mathrm{D} 2)$ & Satt186 & Satt186-Satt226 & 54.88 & 2011Harbin & 8.87 & 16.67 \\
\hline & qIFSF_1 & $13(\mathrm{~F})$ & Satt569 & Satt569-Satt423 & 6.97 & 2011Harbin & 3.09 & 15.84 \\
\hline & qIFSF_2 & $13(\mathrm{~F})$ & Sat_234 & Sat_234-Satt149 & 56.01 & 2011Harbin & 10.92 & 17.89 \\
\hline & dqIFSH_1 & $12(\mathrm{H})$ & Satt302 & Satt302-Satt279 & 0.01 & 2011Harbin & 3.23 & 7.27 \\
\hline & ${ }^{d}$ qIFSL_1 & $19(\mathrm{~L})$ & Sat_134 & Sat_134-Satt278 & 20.99 & 2011Harbin & 7.26 & 16.12 \\
\hline & ${ }^{d}$ qIFSL_2 & $19(\mathrm{~L})$ & Sct_010 & Sct_010-Sat_134 & 43.95 & 2011Harbin & 9.75 & 17.97 \\
\hline & qIFSN_1 & 03(N) & Satt152 & Satt152-Satt530 & 6.67 & 2011Harbin & 2.50 & 27.42 \\
\hline & qIFSN_2 & 03(N) & Satt530 & Satt530-Satt152 & 29.53 & 2011Harbin & 2.50 & 12.80 \\
\hline & dqIFSO_1 & $10(0)$ & Satt345 & Satt345-Satt592 & 6.00 & 2011Harbin & 9.43 & 19.43 \\
\hline & dqIFSO_2 & $10(0)$ & Sat_341 & Sat_341-Satt585 & 88.39 & 2011Harbin & 9.78 & 15.69 \\
\hline \multirow[t]{11}{*}{$\mathrm{F} 3 \mathrm{H}$} & ${ }^{d} \mathrm{qF} 3 \mathrm{HC2}$ _1 & $06(C 2)$ & Satt322 & Satt322-Satt658 & 57.64 & 2011Harbin & 2.27 & 2.37 \\
\hline & qF3HDlb_1 & 02(Dlb) & Satt157 & Satt157-Satt271 & 25.71 & 2011 Harbin & 3.62 & 10.01 \\
\hline & daF3HDlb_2 & 02(Dlb) & Sat_135 & Sat_135-Sat_096 & 30.28 & 2011Harbin & 7.53 & 14.32 \\
\hline & qF3HDlb_3 & 02(Dlb) & Sat_069 & Sat_069-Sat_279 & 168.62 & 2011Harbin & 2.41 & 8.49 \\
\hline & daF3HDlb_4 & 02(Dlb) & Satt459 & Satt459-Sat_069 & 185.58 & 2011Harbin & 2.18 & 5.54 \\
\hline & ${ }^{d} \mathrm{qF} 3 \mathrm{HD} 2 \_1$ & $17(\mathrm{D} 2)$ & Satt031 & Satt031-Sat_326 & 0.01 & 2011Harbin & 2.67 & 1.05 \\
\hline & ${ }^{d} \mathrm{qF} 3 \mathrm{HE}_{-} 1$ & $15(\mathrm{E})$ & Sat_112 & Sat_112-Sat_380 & 22.09 & 2011Harbin & 2.10 & 4.85 \\
\hline & ${ }^{d} \mathrm{qF} 3 \mathrm{HF}_{-} 1$ & $13(F)$ & Sat_262 & Sat_262-Sat_103 & 101.22 & 2011Harbin & 2.63 & 2.24 \\
\hline & dqF3HK_1 & $09(\mathrm{~K})$ & Satt349 & Satt349-Satt518 & 141.56 & 2011Harbin & 2.08 & 1.57 \\
\hline & dqF3HN_1 & 03(N) & Sat_084 & Sat_084-Sat_304 & 41.45 & 2011Harbin & 4.70 & 6.10 \\
\hline & dqF3HO_1 & $10(0)$ & Satt592 & Satt592-Satt633 & 27.54 & 2011Harbin & 2.62 & 2.32 \\
\hline
\end{tabular}

aQTL: The nomenclature of the eQTL included four parts: QTL, trait, linkage group name and QTL order in the linkage group, respectively.

${ }^{b}$ Position from the left marker of the interval on each linkage group.

'Proportion of phenotypic variance $\left(R^{2}\right)$ explained by a eQTL.

${ }^{\mathrm{d}}$ Trans-eQTL, others are cis-eQTL.

Identification of candidate genes underlying the overlapped loci of pQTL and eQTL

Thirty four pQTLs for both individual and total seed isoflavone contents of soybean were compared with eQTLs to identify the overlapped loci. Five eQTL intervals were overlapped with pQTLs, and a total of eleven candidate genes within the overlapped eQTL and PQTL were identified (Table 4). Two genes, C4H (Glyma02g40290.1) and PAL1 (Glyma02g47940.1), were identified on Gm02 (LG
D1b) between Satt546-Satt459. CHI (Glyma17g34430.1) and DFR (dihydroflavonol reductase; EC 1.1.1.219) were identified on Gm17 (LG D2) between Satt186-Satt226. Genes encoding 4-coumarate-CoA ligase (EC 6.2.1.12; Glyma13g01080.1/2), FLS (Glyma13g02740.1) and CHS (Glyma13g09640.1) were identified on Gm13 (LG F) between Satt423-Satt569. Additionally, CHS (Glyma13g24200.1) and IFS (Glyma13g09640.1) was found within another eQTL/pQTL interval (Satt149-Sat_234). 


\section{Discussion}

Soybean isoflavones have been broadly used in food, medicine, cosmetics and animal husbandry [59]. Increasing and decreasing seed isoflavone content will be an important target of soybean breeding. MAS based on genotype selection rather than solely on phenotype selection provides additional power for the selections during soybean breeding [60]. Cultivar 'Zhongdou 27' proved to have highisoflavone content $(3,791 \mu \mathrm{g} / \mathrm{g}$ isoflavone in seed) as reported previously [16]. Meng et al. [19] identified two QTL underlying resistance to soybean aphid through leaf isoflavone-mediated antibiosis in soybean cultivar 'Zhongdou 27'. A number of pQTLs associated with seed isoflavone were identified in multiple environments from cultivar 'Zhongdou 27' using 194 SSR markers (accepted by Molecular Biology Reports). Therefore, 'Zhongdou 27' should be given more attention as an elite germplasm to improve soybean seed isoflavone concentration, disease and pest resistances.

In our previous studies, some identified QTLs associated with individual/total isoflavone contents showed higher contribution to phenotypic variation. Some specific copies of genes (PAL, CHS, IFS, F3H) in the phenylpropanoid pathway were near or falling into these quantitative trait loci by browsing the reference genome sequence of Williams 82 (http://www.phytozome.net/soybean).

To investigate the regulation mechanism of isoflavone synthetic enzyme genes, the transcript abundances of $P A L$, $C H S$, IFS and $F 3 H$ in the mapping population were examined, and the genomic regions affecting the expression of 
Table 4 Identification of candidate genes underlying overlapped locus of eQTL and QTL

\begin{tabular}{|c|c|c|c|c|c|}
\hline $\begin{array}{l}\text { Marker } \\
\text { interval }\end{array}$ & $\mathrm{Gm}(\mathrm{LG})$ & $\begin{array}{c}\text { Physical location } \\
\text { of markers }\end{array}$ & Candidate genes & $\begin{array}{l}\text { Physical location of } \\
\text { candidate genes }\end{array}$ & $\begin{array}{c}\text { Function of candidate } \\
\text { genes }\end{array}$ \\
\hline \multirow[t]{2}{*}{ Satt546-Satt459 } & Gm02(LGD1b) & $43,775,407-48,390,089$ & Glyma02g40290.1 & $45,490,798-45,495,043$ & $\mathrm{C} 4 \mathrm{H}$ \\
\hline & & & Glyma02g47940.1 & $51,366,326-51,368,943$ & PAL1 \\
\hline \multirow[t]{2}{*}{ Satt186-Satt226 } & Gm17(LG D2) & $26,768,866-39,047,375$ & Glyma17g34430.1 & $38,398,978-38,401,025$ & $\mathrm{CHI}$ \\
\hline & & & Glyma17g37060.1 & $40,920,379-40,923,898$ & $D F R$ \\
\hline \multirow[t]{3}{*}{ Satt423-Satt569 } & Gm13(LG F) & $5,231,035-9,567,285$ & Glyma13g01080.1/2 & $798,836-805,844$ & $4 C L$ \\
\hline & & & Glyma13g02740.1 & $2,707,784-2,712,790$ & $F L S$ \\
\hline & & & Glyma13g09640.1 & $11,153,569-11,158,812$ & CHS \\
\hline \multirow[t]{5}{*}{ Satt149-Sat_234 } & Gm13(LG F) & $4,976,740-26,460,745$ & Glyma13g24200.1 & $27,567,360-27,569,061$ & IFS \\
\hline & & & Glyma13g20800.1 & $24,273,025-24,278,037$ & PAL1 \\
\hline & & & Glyma13g27380.1 & $30,577,113-30,579,230$ & $D F R$ \\
\hline & & & Glyma13g09640.1 & $11,153,569-11,158,812$ & CHS \\
\hline & & & Glyma13g02740.1 & $2,707,784-2,712,790$ & $F L S$ \\
\hline \multirow[t]{4}{*}{ Sat_262-Sat_103 } & Gm13(LG F) & $7,233,012-25,478,474$ & Glyma13g20800.1 & $24,273,025-24,278,037$ & PAL1 \\
\hline & & & Glyma13g24200.1 & $27,567,360-27,569,061$ & IFS \\
\hline & & & Glyma13g09640.1 & $11,153,569-11,158,812$ & $\mathrm{CHS}$ \\
\hline & & & Glyma13g02740.1 & $2,707,784-2,712,790$ & $F L S$ \\
\hline
\end{tabular}

the TGs were identified using the eQTL methodology [61]. A global microarray eQTL analysis of a limited number of samples can be used for exploring functional and regulatory gene networks and for scanning cis-eQTL, whereas the subsequent analysis of a subset of likely cis-regulated genes by real-time RT-PCR in a larger number of samples may identify QTL region by targeting these positional candidate genes [62]. In this study, real-time PCR reactions were used to analyze the transcript abundance variations of the four TGs in the $\mathrm{F}_{5: 11}$ RI lines.

When combined with classical QTL phenotypes, correlation analysis can directly provide an overview of potential genes underlying isoflavone traits [63,64]. Through the comparison of the transcript abundances of the four TGs (PAL, CHS, IFS and F3H), the parents ('Zhongdou 27' and 'Jiunong 20') showed different patterns at the R6 stage. This observation was consistent with the previous report by Sarah et al. [65]. Significant correlations between the transcript abundances of TGs and isoflavone contents were found in developing seeds at the R6 stage, indicating that these genes could affect total and individual isoflavone accumulations (Table 2).

Previously, two major QTLs that affect isoflavone content across multiple environments were mapped on $\mathrm{Gm} 05$ (LG A1) and Gm08 (LG A2) by Gutierrez et al. [17] and Yang et al. [20], respectively. In the present work, one eQTL qIFSA2_1 (Sat_129-Sat_181) was mapped close to qGCA2_1 on Gm08 (LG A2) (Figure 1, Table 5). This result suggested that qIFSA2_1 might be a cis-enzyme related locus. Some of these identified eQTLs associated with seed isoflavone content did not coincide with the
TGs, suggesting that the differences in TGs transcript abundances might be caused by several trans-acting factors [66].

In this study, since the 194 markers were not uniformly distributed, large gaps appeared with low marker density on chromosomes Gm02, 04, 13, 16 and 18, implying that more markers should be developed among these gaps and the authenticity of pQTL or eQTL should be further clarified. Among these gaps, special attention should be paid to eQTL qF3HDlb_2 on chromosome Gm02 and qIFSC1_1 on chromosome Gm04 because of their higher LOD score and contribution to phenotypic variation (Table 3). Overlapped loci of qF3HF_1 and qDZF_1, and genes that fall into this region should also be further clarified with more markers. Consequently, fine mapping on these intervals with more SSR or SNP markers and to determine the authenticity of these loci as well as the underlying genes were extremely essential in the future work.

The analysis of eQTL overlapped with PQTL suggested that the candidate genes or elements among the marker intervals could affect phenotypic traits $[49,67,68]$. Therefore, overlapped loci of eQTLs and pQTLs were analyzed to find the potential candidate genes affecting the accumulation of isoflavone contents in soybean seed. Five eQTL intervals were overlapped with pQTLs according to the comparison of genomic regions between pQTLs and eQTLs (Table 5). These results indicated that some candidate genes or elements in these intervals could regulate the biosynthesis of isoflavone components, and affect their accumulation. Additionally, some eQTLs overlapped with 
Table 5 Partial QTLs for individual and total isoflavone contents

\begin{tabular}{|c|c|c|c|c|c|c|c|c|}
\hline Traits $^{a}$ & $\mathrm{QTL}^{\mathrm{b}}$ & Gm (LG) & Marker & Marker interval & Position $^{c}$ & Environment $^{d}$ & LOD score & $R^{2}(\%)^{e}$ \\
\hline DZ & $f_{\text {fDZF_1 }}$ & $13(\mathrm{~F})$ & Sat_103 & Sat_103-Sat_262 & 188.34 & E2 & 2.00 & 10.57 \\
\hline \multirow[t]{3}{*}{ GC } & qGCA2_1 & $08(A 2)$ & Sat_040 & Sat_040-Satt233 & 38.46 & E3 & 2.65 & 6.01 \\
\hline & ${ }^{f} \mathrm{qGCD} 1 \mathrm{~b} \_1$ & 02(Dlb) & Satt546 & Satt546-Sat_459 & 215.67 & E2 & 2.38 & 3.12 \\
\hline & & & & & & E5 & 2.21 & 4.17 \\
\hline \multirow[t]{7}{*}{ GT } & fqGTD2_1 & $17(\mathrm{D} 2)$ & Satt186 & Satt186-Satt226 & 50.81 & E1 & 2.00 & 3.41 \\
\hline & & & & & & E2 & 2.36 & 5.23 \\
\hline & & & & & & E3 & 5.76 & 10.98 \\
\hline & & & & & & E5 & 3.09 & 8.23 \\
\hline & fqGTF_2 & $13(\mathrm{~F})$ & Satt149 & Satt149-Sat_234 & 41.23 & E1 & 2.00 & 1.56 \\
\hline & & & & & & E3 & 2.49 & 4.17 \\
\hline & & & & & & E7 & 4.03 & 5.47 \\
\hline \multirow[t]{2}{*}{$\mathrm{Tl}$} & ${ }_{\text {qTTF_1 }}$ & $13(\mathrm{~F})$ & Satt423 & Satt423-Satt569 & 6.01 & E6 & 4.59 & 3.21 \\
\hline & & & & & & E7 & 2.15 & 4.2 \\
\hline
\end{tabular}

${ }^{\mathrm{a} D Z}$ : Daidzein; GC:Glycitein; GT: Genistein; TI: Total isoflavone.

${ }^{b}$ The nomenclature of the QTL included four parts : QTL, trait, linkage group name and QTL order in the linkage group, respectively.

${ }^{C}$ Position from the left marker of the interval on each linkage group.

dE1: at Harbin in 2005, E2: at Harbin in 2006, E3: at Hulan in 2006, E4:at Suihua in 2006, E5: at Harbin in 2007, E6: at Hulan in 2007 , E7: at Suihua in 2007.

eProportion of phenotypic variance (R2) explained by a QTL.

fOverlapped loci of $\mathrm{pQTL}$ and $\mathrm{eQTL}$.

other eQTLs or shared the same markers with pQTLs, suggesting that some candidate genes or elements were located near these loci.

Several genes involved in isoflavone accumulation in soybean seed had been identified [22,27,31]. 11 candidate genes falling into the overlapped intervals of $\mathrm{pQTL}$ and eQTL were found (Table 4). Bolon et al. [58] identified eQTL for genes with seed-specific expression and discovered striking eQTL hotspots at distinct genomic intervals on chromosome Gm13. A chalcone isomerase (CHI3) and IFS2 gene were located in the same region identified by qGEN13 on Gm13 [11]. Another QTL for GC that encoded $P A L$ and $4 C L$ paralog was also reported on Gm13 [10,11]. In the present work, seven candidate genes on Gm13 (LG F) were identified, implying that there could be a hotspot of gene cluster that regulated seed isoflavone content on Gm13. Among them, CHS (Glyma13g09640.1) and FLS (Glyma13g02740.1) were identified on three overlapped loci, implying that they could interact or trans-regulate other genes in the phenylpropanoid pathway. Furthermore, PAL1 (Glyma13g20800.1) and IFS (Glyma13g24200.1) paralogs were identified within two overlapped loci. In the marker interval (Satt149-Sat_234) associated with qCHSF_1, qIFSF_2 and qGTF_2, both Glyma13g24200.1 and Glyma13g09640.1 were found to encode CHS and IFS, indicating that they could be the potential candidate genes. It was supposed that Glyma13g09640.1 could interact or trans-regulate the expression of IFS. However, the function of these potential candidate genes should be tested in future works.
Although open questions about the biology and applications of eQTL mapping still exist [69], there are considerable advances in the eQTL studies. Detailed analysis of eQTL combined with cluster analysis of transcript abundance and eventually gene expression patterns could assist map-based cloning of genes underlying these traits. Markers based on underlying genes are also desirable for MAS in soybean breeding programs. The mechanism underlying seed isoflavone synthesis and its accumulation may contribute to the development of marker-assisted selection for soybean cultivars with high or low isoflavone contents.

\section{Conclusions}

A total of thirty three eQTLs (thirteen cis-eQTLs and twenty trans-eQTLs) were identified on fifteen chromosomes. Five eQTL intervals were overlapped with pQTLs and a total of eleven candidate genes within the overlapped eQTL and pQTL were identified. These results might be beneficial for the development of markerassisted selection to breed soybean cultivars with high isoflavone contents.

\section{Methods}

Plant materials and growing conditions

The mapping population of $130 \mathrm{~F}_{5: 11}$ recombinant inbred (RI) lines were derived through single-seed-descent from the cross between 'Zhongdou 27' (developed by the Chinese Academy of Agricultural Sciences, Beijing, China) and 'Jiunong 20' (developed by Jilin Academy of Agricultural Sciences, Jilin, China). 'Zhongdou 27' contains high 
individual and total isoflavone (TI) contents in seed (daidzein, DZ, 1,865 $\mu \mathrm{g} / \mathrm{g}$; genistein, GT, 1,614 $\mu \mathrm{g} / \mathrm{g}$; glycitein, $\mathrm{GC}, 311 \mu \mathrm{g} / \mathrm{g}$ and total isoflavone, TI, 3,791 $\mu \mathrm{g} / \mathrm{g}$ ), whereas 'Jiunong 20' has low individual and TI contents (DZ, $844 \mu \mathrm{g} / \mathrm{g} ; \mathrm{GT}, 1,046 \mu \mathrm{g} / \mathrm{g} ; \mathrm{GC}, 193 \mu \mathrm{g} / \mathrm{g}$ and TI, $2,061 \mu \mathrm{g} / \mathrm{g})$.

To detect eQTL, the parents and the $130 \mathrm{~F}_{5: 11}$ RI lines were planted at Harbin, Heilongjiang Province, China, in 2011. Randomized complete block designs were used for all experiments with rows $3 \mathrm{~m}$ long, $0.65 \mathrm{~m}$ apart, and a space of $6 \mathrm{~cm}$ between plants. Mature and immature seeds in the reproductive stages (from soybean growth stage R3 to R8) [70] were harvested from a bulked sample collected from three plants in each plot. These samples were quantified for individual and total seed isoflavone contents and transcript abundances.

\section{Isoflavone extraction and quantification}

Approximately $150 \mathrm{~g}$ of soybean seed samples were ground to a fine power using a commercial coffee grinder. Isoflavones were extracted from flour and separated using HPLC as described previously [16]. Measurements were done as micrograms of isoflavone per gram of seeds plus and minus the standard deviations $(\mu \mathrm{g} / \mathrm{g} \pm \mathrm{SD})$.

\section{Synthesis of cDNA, Real-Time PCR and data collection}

To investigate the expressions of four TGs, total RNA was isolated from soybean seed samples from R3 to R8 stages using plant RNA purification reagent Kit (D9108A, TaKaRa, Japan). RNAs were transcribed to cDNA using the first strand DNA synthesis reagent Kit (D6110A, TaKaRa, Otsu, Shiga, Japan). Four TGs (PAL, GenBank accession: GQ220305; CHS, GenBank accession: EU526827; IFS, GenBank accession: FJ770473 and F3H, GenBank accession: AY595420) in the phenylpropanoid pathway, were selected to analyze the transcript abundance variations in the $F_{5: 11}$ RI line population. These four TGs were analyzed by real-time PCR (Kit DRR081A, TaKaRa, Japan). Genespecific primers for expression analysis of the four TGs were listed in Table 6. Primer specificity was confirmed based on each primer pair sequence against soybean genome sequences by BLASTing (http://www.phytozome.net/ soybean) using the BLASTN algorithm. Moreover, through the BLASTN of the sequences of the TGs, PAL2 (located on Gm10 (LG O)) of the PAL gene family, CHS8 (located on Gm11 (LG B1)) of the CHS gene family, IFS1 (located on $\mathrm{Gm07}$ (LG M)) of the IFS gene family, and F3H1 and F3H2 (located on Gm02 (LG D1b)) of the F3H gene family were amplified [11].

PCR amplification was performed as follows: $95^{\circ} \mathrm{C}$ for $60 \mathrm{~s}$, followed by 40 cycles of $95^{\circ} \mathrm{C}$ for $11 \mathrm{~s}, 60^{\circ} \mathrm{C}$ for $12 \mathrm{~s}$ and $72^{\circ} \mathrm{C}$ for $18 \mathrm{~s}$. The soybean actin4 (GenBank accession: AF049106) gene was used as a reference to quantify the expression levels of the target genes [71]. Three replicates for each reaction were performed. The relative transcript abundance of TGs in different samples was calculated using $2^{-\Delta \Delta \mathrm{Ct}}$ method [72], defined as: $\Delta \mathrm{Ct}=\mathrm{Ct}$ (target) $-\mathrm{Ct}$ (actin). Pearson correlations between total/individual isoflavone contents and the expression of the four TGs in $\mathrm{F}_{5: 11}$ RILs were evaluated using SAS 8.2 (Cary, NC, USA) [73].

\section{Identification of genomic region of target genes}

The whole genome sequence Glyma1 assembly for Williams 82 [74] provided a powerful tool for interrogating QTL data. Previously reported genes for isoflavone biosynthesis [75] were used in BLAST searches against the whole genome sequence to identify homologous regions in the genome with assigned or putative functions. All twenty soybean chromosomes have regions sharing a high percentage of homology with genes of known function in the phenylpropanoid pathway [11]. The coding regions of TGs were compared with genome of Williams 82 through BLAST (E-value $\leq 1.0 \mathrm{E}-05$, http://www.phytozome.net/soybean) to identify homologous regions.

\section{eQTL analysis}

In previous work, fifteen QTL underlying seed isoflavone contents of soybean were identified based on RI line populations derived from a cross between 'Zhongdou 27' (high isoflavone) and 'Jiunong 20' (low isoflavone) through a genetic linkage map including 99 SSR markers [16]. Another 95 SSR markers were added to the map of Zeng et al. [16] to identify novel phenotypic QTLs (pQTLs) associated with seed isoflavone contents of soybean (accepted by Molecular Biology Reports). In this study, 194 polymorphic markers were assembled onto the 20 linkage groups (LGs) by Mapmaker 3.0b with the Kosambi mapping function [76]. WinQTLCart2.1 [77] was used to detect eQTL between marker intervals by 1,000 permutations at significance $(P \leq 0.05)$. The genetic linkage map was

Table 6 Real-time PCR primer pairs for the expression analyses of $P A L, C H S, F 3 H$, and IFS genes

\begin{tabular}{lll}
\hline Gene & Forward primer $\mathbf{( 5 ' - 3 ' )}$ & Reverse primer (5'-3') \\
\hline Actin4 & GTGTCAGCCATACTGTCCCCATT & GTTCAAGCTCTTGCTCGTAATCA \\
PAL & ATTATGGATTCAAGGGAGT & AATGAGGAAAGTGAGGACA \\
CHS & AAAATGCCATCTCCTCAAACA & GGATCTCAGCTACGCTCACC \\
F3H & GCTTGCGAGATTGGGGTAT & CCTTGGAGATGGCTGGAGAC \\
IFS & GCCCTGGAGTCAATCTGG & CAAGACTATGTGCCCTGGA \\
\hline
\end{tabular}


constructed using Mapchart 2.1 [78]. The nomenclature of the eQTLs/pQTLs included four parts following the recommendations of the Soybean Germplasm Coordination Committee. For example, qCHSF_1, q, CHS, F and 1 represent eQTL, trait $(C H S)$, linkage group name and eQTL order in the linkage group, respectively.

\section{Identification of candidate genes underlying overlapped loci of PQTL and eQTL}

Coincident genetic locations of eQTL and pQTL may be available to identify important regulatory genes underlying traits, and lead to the identification of molecular mechanisms $[49,67,68]$. Previous studies have combined eQTL and PQTL mapping to gain insight into regulatory pathways involved in determining phenotypic traits [49,68,79-81]. eQTL located in the same marker intervals of pQTL might contribute to significant phenotypic variations $[49,67,68]$. In this study, thirty four phenotypic QTL (pQTL) identified with the 194 SSR markers were compared with eQTL to identify overlapped loci. Genetic map positions were estimated by identifying the nearest flanking SSR markers using the genome browser (http://www. soybase.org). The candidate genes underlying overlapped loci of PQTL and eQTL were identified by browsing after using BLAST search of flanking markers against the whole genome sequence of Williams 82 (available at: http:// www.phytozome.net/soybean).

\section{Competing interests}

The authors declare that they have no competing interests.

\section{Authors' contributions}

WL conceived the project and its components, contributed to the original concept of the project. YW and YH performed the SSR marker screening, eQTL analyses and paper writing. WT, XZ, YL, LW and DL collected samples and performed the phenotype analyses. All authors read and approved the final manuscript

\section{Acknowledgements}

This study was conducted in Soybean Research \& Development Center (CARS) and Collaborative Innovation Center of Grain Production Capacity Improvement in Heilongjiang Province, financially supported by National Core Soybean Genetic Engineering Project (2014ZX08004-003), National 863 Project (2013AA102602), National 973 Project (2012CB126311), National 863 Project (2012AA101106-1-9), and Provincial/National Education Ministry for the team of soybean molecular design.

Received: 29 April 2014 Accepted: 30 July 2014

Published: 13 August 2014

\section{References}

1. Tsukamoto C, Shimada S, Igita K, Kudou S, Kokubun M, Okubo K, Kitamura K: Factors affecting isoflavone content in soybean seeds: Changes in isoflavones, saponins, and composition of fatty acids at different temperatures during seed development. J Agric Food Chem 1995, 43:1184-1192.

2. Munro IC, Harwood M, Hlywka JJ, Stephen AM, Doull J, Flamm WG, Adlercreutz H: Soy isoflavones: a safety review. Nutr Rev 2003, 61:1-33.

3. Cederroth $\mathrm{CR}$, Nef S: Soy, phytoestrogens and metabolism: a review. Mol Cell Endocrinol 2009, 304:30-42.

4. Benhamou N, Nicole M: Cell biology of plant immunization against microbial infection: the potential of induced resistance in controlling plant diseases. Plant Physiol Biochem 1999, 37:703-719.
5. Subramanian S, Graham MY, Yu O, Graham TL: RNA interference of soybean isoflavone synthase genes leads to silencing in tissues distal to the transformation site and to enhanced susceptibility to Phytophthora sojae. Plant Physiol 2005, 137:1345-1353.

6. Subramanian S, Stacey G, Yu O: Distinct, crucial roles of flavonoids during legume nodulation. Trends Plant Sci 2007, 12:282-285.

7. Lozovaya W, Lygin AV, Zernova OVLISX, Hartman GL, Widholm M: Isoflavonoid accumulation in soybean hairy roots upon treatment with Fusarium solani. Plant Physiol Biochem 2004, 42:671-679.

8. Meksem K, Njiti VN, Banz WJ, Iqbal MJ, Kassem MM, Hyten DL, Yuang f, Winters TA, Lightfoot DA: Genomic regions that underlie soybean seed isoflavone content. J Biomed Biotechnol 2001, 1(1):38-44.

9. Kassem MA, Meksem K, lqbal MJ, Njiti VN, Banz WJ, Winters TA, Wood A, Lightfoot DA: Definition of soybean genomic regions that control seed phytoestrogen amounts. J Biomed Biotechnol 2004, 1:52-60.

10. Primomo VS, Poysa V, Ablett GR, Jackson CJ, Gijzen M, Rajcan I: Mapping QTL for individual and total isoflavone content in soybean seeds. Crop Sci 2005, 45:2454-2462.

11. Gutierrez GJJ, Wu XL, Gillman JD, Lee JD, Zhong R, Yu O, Shannon G, Ellersieck M, Nguyen HT, Sleper DA: Intricate environment-modulated genetic networks control isoflavone accumulation in soybean seeds. BMC Plant Biol 2010, 10:105-120.

12. Hoeck JA, Fehr WR, Murphy PA, Welke GA: Influence of genotype and environment on isoflavone contents of soybean. Crop Sci 2000, 40:48-51.

13. Dhaubhadel S, McGarvey BD, Williams R, Giizen M: Isoflavonoid biosynthesis and accumulation in developing soybean seeds. Plant $\mathrm{Mol}$ Biol 2003, 53:733-743.

14. Murphy SE, Lee EA, Woodrow L, Seguin P, Kumar J, Rajcan I, Ablett GR: Genotype $\times$ Environment interaction and stability for isoflavone content in soybean. Crop Sci 2009, 49:1313-1321.

15. Kassem MA, Shultz J, Meksem K, Cho Y, Wood AJ, lqbal MJ, Lightfoot DA: An updated 'Essex' by 'Forrest' linkage map and first composite interval map of QTL underlying six soybean traits. Theor Appl Genet 2006, 113:1015-1026.

16. Zeng G, Li D, Han Y, Teng W, Wang J, Qiu L, Li W: Identification of QTL underlying isoflavone contents in soybean seeds among multiple environments. Theor App/ Genet 2009, 118:1455-1463.

17. Gutierrez GJJ, Vuong TD, Zhong R, Yu O, Lee JD, Shannon G, Ellersieck M, Nguyen HT, Sleper DA: Major locus and other novel additive and epistatic loci involved in modulation of isoflavone concentration in soybean seeds. Theor Appl Genet 2011, 123:1375-1385.

18. Liang HZ, Yu YL, Wang SF, Lian Y, Wang TF, Wei YL, Gong PT, Liu XY, Fang $X J$, Zhang MC: QTL mapping of isoflavone, oil and protein contents in soybean (Glycine max L. Merr.). Agric Sci China 2010, 9:1108-1116.

19. Meng FL, Han YP, Teng WL, Li YG, Li WB: QTL underlying the resistance to soybean aphid (Aphis glycines Matsumura) through isoflavone-mediated antibiosis in soybean cultivar 'Zhongdou 27'. Theor Appl Genet 2011, 123:1459-1465,

20. Yang K, Moon JK, Jeong N, Chun HK, Kang ST, Back K, Jeong SC: Novel major quantitative trait loci regulating the content of isoflavone in soybean seeds. Genes Genom 2011, 33:685-692.

21. Zhang JY, Ge YN, Sun JM, Han FX, Yu FK, Yan SR, Yang H: Identification of QTLs for major isoflavone components among multiple environments in soybean seeds. Sci Agric Sin 2012, 45:3909-3920.

22. Akond M, Richard B, Ragin B, Herrera H, Kaodi U, Akbay C, Kantartzi SK, Njiti V, Barakat A, Meksem K, Lightfoot DA, Kassem MA: Additional quantitative trait loci and candidate genes for seed isoflavone content in soybean. J Agric Sci 2013, 5:20-33.

23. Akond M, Liu SM, Kantartzi SK, Meksem K, Bellaloui N, Lightfoot DA, Yuan JZ, Wang DC, Kassem MA: Quantitative trait loci for seed isoflavone contents in 'MD96-5722' by 'Spencer' recombinant inbred lines of soybean. J Agric Food Chem 2014, 62:1464-1468.

24. Wellmann E: UV dose-dependent induction of enzymes related to flavonoid biosynthesis in cell suspension cultures of parsley. FEBS Lett 1975, 51:105-107.

25. Elio GWM, CH, Arjen J, Arnaud G: Modification of flavonoid biosynthesis in crop plants. Phytochemistry 2004, 65:2631-2648.

26. Austin MB, Noel JP: The chalcone synthase superfamily of type III polyketide synthases. Nat Prod Rep 2003, 20:79-110.

27. Du H, Huang YB, Tang YX: Genetic and metabolic engineering of isoflavonoid biosynthesis. Appl Microbiol Biotechnol 2010, 86:1293-1312. 
28. Forkmann G, Martens S: Metabolic engineering and applications of flavonoids. Curr Opin Biotechnol 2001, 12:155-160.

29. Joung JY, Kasthuri M, Park JY, Kang WJ, Kim HS, Yoon BS, Joung H, Jeon JH: An overexpression of chalcone reductase of Pueraria montana var. lobata alters biosynthesis of anthocyanin and 50-deoxyflavonoids in transgenic tobacco. Biochem Biophys Res Commun 2003, 3003:326-331.

30. Jung W, Yu O, Lau SC, O'Keefe DP, Odell J, Fader G, McGonigle B: Identification and expression of isoflavone synthase, the key enzyme for biosynthesis of isoflavones in legumes. Nat Biotechnol 2000, 18:208-212.

31. Hao C, Oliver Y, Deyue Y: Polymorphisms of IFS1 and IFS2 gene are associated with isoflavone concentrations in soybean seeds. Plant Sci 2008, 175:505-512

32. Britsch L, Dedio J, Saedler H, Forkmann G: Molecular characterization of flavanone 3 beta-hydroxylases. Consensus sequence, comparison with related enzymes and the role of conserved histidine residues. Eur 」 Biochem 1993, 217:745-754

33. Cheng H, Wang J, Chu S, Yan H-L, Yu D: Diversifying selection on flavanone 3-hydroxylase and isoflavone synthase genes in cultivated soybean and its wild progenitors. PLoS One 2013, 8:e54154.

34. Yu O, McGonigle B: Metabolic engineering of isofavone biosynthesis. Adv Agron 2005, 86:147-190.

35. Yu O, Shi J, Hession AO, Maxwell CA, McGonigle B, Odell JT: Metabolic engineering to increase isoflavone biosynthesis in soybean seed. Phytochemistry 2003, 63:753-763.

36. Yin Z, Meng F, Song H, Wang X, Xu X, Yu D: Expression quantitative trait loci analysis of two genes encoding RUBISCO activase in soybean. Plant Physiol 2010, 152:1625-1637.

37. Jansen R, Nap J: Genetical genomics: the added value from segregation Trends Genet 2001, 17:388-391.

38. Cheung VG, Conlin LK, Weber TM, Arcaro M, Jen KY, Morley M, Spielman RS: Natural variation in human gene expression assessed in lymphoblastoid cells. Nat Genet 2003, 33:422-425.

39. Goring HH, Curran JE, Johnson MP, Dyer TD, Charlesworth J, Cole SA, Jowett JBM, Abraham LJ, Rainwater DL, Comuzzie AG, Mahaney MC, Almasy L, MacCluer JW, Kissebah AH, Collier GR, Moses EK, Blangero J: Discovery of expression QTLs using large-scale transcriptional profiling in human lymphocytes. Nat Genet 2007, 39:1208-1216.

40. Sasayama D, Hori H, Nakamura S, Miyata R, Teraishi T, Hattori K, Ota M, Yamamoto N, Higuchi T, Amano N, Kunugi H: Identification of single nucleotide polymorphisms regulating peripheral blood mRNA expression with Genome-Wide Significance: an eQTL study in the Japanese population. PLoS One 2013, 8:e54967.

41. DeCook R, Lall S, Nettleton D, Howell SH: Genetic regulation of gene expression during shoot development in Arabidopsis. Genetics 2006 172:1155-1164.

42. Jordan MC, Somers DJ, Banks TW: Identifying regions of the wheat genome controlling seed development by mapping expression quantitative trait loci. Plant Biotechnol J 2007, 5:442-453.

43. Potokina E, Druka A, Luo Z, Wise R, Waugh R, Kearsey M: Gene expression quantitative trait locus analysis of 16,000 barley genes reveals a complex pattern of genome-wide transcriptional regulation. Plant J 2008, 53:90-101.

44. Chen X, Hackett CA, Niks RE, Hedley PE, Booth C, Druka A, Marcel TC, Vels A, Bayer M, Milne I, Morris J, Ramsay L, Marshall D, Cardle L, Waugh R: An eQTL analysis of partial resistance to Puccinia hordeii in barley. PLoS One 2010, 5(1):e8598

45. Brem RB, Yvert G, Clinton R, Kruglyak L: Genetic dissection of transcriptional regulation in budding yeast. Science 2002, 296:752-755.

46. Brem RB, Kruglyak L: The landscape of genetic complexity across 5,700 gene expression traits in yeast. Proc Natl Acad Sci U S A 2005, 102:1572-1577.

47. Li Y, Alvarez OA, Gutteling EW, Tijsterman M, Fu J, Riksen JAG, Hazendonk E, Prins P, Plasterk RHA, Jansen RC, Breitling R, Kammenga JE: Mapping determinants of gene expression plasticity by genetical genomics in $C$. elegans. PLoS Genet 2006, 2:2155-2161.

48. Hughes KA, Ayroles JF, Reedy MM, Drnevich JM, Rowe KC, Ruedi EA, Caceres CE, Paige KN: Segregating variation in the transcriptome: cis regulation and additivity of effects. Genetics 2006, 173:1347-1355.

49. Schadt EE, Monks SA, Drake TA, Lusis AJ, Che N, Colinayo V, Ruff TG, Milligan SB, Lamb JR, Cavet G, Linsley PS, Mao M, Stoughton RB, Friend SH: Genetics of gene expression surveyed in maize, mouse and man. Nature 2003, 22:297-302
50. Ghazalpour A, Doss S, Zhang B, Wang S, Plaisier S, Castellanos R, Brozell A, Schadt EE, Drake TA, Lusis AJ, Horvath S: Integrating genetic and net-work analysis to characterize genes related to mouse weight. PLoS Genet 2006, 2:e130.

51. Çinar MU, Fan H, Neuhoff C, Große-Brinkhaus C: eQTL Analysis and association of MYF6 mRNA expression with meat quality traits in pigs. Kafkas Universitesi Veteriner Fakultesi Dergisi 2012, 18:235-242.

52. Hubner N, Wallace CA, Zimdahl H, Petretto E, Schulz H, Maciver F, Mueller M, Hummel O, Monti J, Zidek V, Musilova A, Kren V, Causton H, Game L, Born G, Schmidt S, Muller A, Cook SA, Kurtz TW, Whittaker J, Pravenec M, Aitman TJ: Integrated transcriptional profiling and linkage analysis for identification of genes underlying disease. Nat Genet 2005, 37:243-253.

53. Kliebenstein D: Quantitative Genomics: analyzing intraspecific variation using global gene expression polymorphisms or eQTL. Annu Rev Plant Biol 2008, 60:93-114

54. Doss S, Schadt EE, Drake TA, Lusis AJ: Cis-acting expression quantitative trait loci in mice. Genome Res 2005, 15:681-691.

55. Gatti DM, Harrill AH, Wright FA, Threadgill DW, Rusyn I: Replication and narrowing of gene expression quantitative trait loci using inbred mice. Mamm Genom 2009, 20:437-446.

56. Yin Z, Meng F, Song $H$, Wang X, Chao M, Zhang G, Xu X, Deng D, Yu D: $\mathrm{GmFtsH} 9$ expression correlates with in vivo Photosystem II function: Chlorophyll a fluorescence transient analysis and eQTL mapping in soybean. Planta 2011, 234:815-827.

57. Song $H$, Yin Z, Chao M, Ning L, Zhang D, Yu D: Functional properties and expression quantitative trait loci for phosphate transporter GmPT1 in soybean. Plant Cell Environ 2014, 37:462-472.

58. Bolon YT, Hyten DL, Orf JH, Vance CP, Muehlbauer GJ: eQTL networks reveal complex genetic architecture in the immature soybean seed. Plant Genom 2014, 7:1-14.

59. Brouns F: Soya isoflavones: a new and promising ingredient for the health food sector. Food Res Int 2002, 35:187-193.

60. Bernardo R: Molecular markers and selection for complex traits in plants: learning from the last 20 years. Crop Sci 2008, 48:1649-1664.

61. Sladek R, Hudson TJ: Elucidating cis-and trans-regulatory variation using genetical genomics. Trends Genet 2006, 22:245-250.

62. Ponsuksili S, Murani E, Phatsara C, Schwerin M, Schellander K, Wimmers K: Expression quantitative trait loci analysis of genes in porcine muscle by quantitative real-time RT-PCR compared to microarray data. Heredity 2010, 105:309-317.

63. Druka A, Potokina E, Luo ZW, Bonar N, Druka I, Zhang L, Marshall DF, Steffenson BJ, Close TJ, Wise RP, Kleinhofs A, Williams RW, Kearsey MJ, Waugh R: Exploiting regulatory variation to identify genes and loci underlying quantitative traits in barley. Theor Appl Genet 2008, 117:261-272.

64. Holloway B, Li B: Expression QTLs: applications for crop improvement. Mol Breed 2010, 26:381-391.

65. Sarah IJ, Delkin OG, Lila OV: Flux of transcript patterns during soybean seed development. BMC Genom 2010, 11:136-150.

66. Liu P, Wang CM, Li L, Sun F, Yue GH: Mapping QTL for oil traits and eQTL for oleosin genes in jatropha. BMC Plant Biol 2011, 11:132-140.

67. Wang J, Yu H, Xie W, Xing Y, Yu S, Xu C, Zhang Q: A global analysis of QTLs for expression variations in rice shoots at the early seedling stage. Plant J 2010, 63:1063-1074

68. Chen X, Guo W, Liu B, Zhang Y, Song X, Cheng Y, Zhang L, Zhang T: Molecular mechanisms of fiber differential development between $\mathrm{G}$. barbadense and $\mathrm{G}$. hirsutum revealed by genetical genomics. PLoS One 2012, 7:e30056.

69. Gilad Y, Rifkin SA, Pritchard JK: Revealing the architecture of gene regulation: the promise of eQTL studies. Trends Genet 2008, 24:408-415.

70. Fehr WR, Caviness CE: Stages of Soybean Development. In Special Report 80, Cooperative Extension Service, Agriculture and Home Economic Experiment Station. Ames, lowa: lowa State University; 1977:1-11.

71. Iqbal MJ, Yaegashi S, Njiti VN, Ahsan R, Cryder KL, Lightfoot DA: Gene locus pyramids alter transcript abundance in soybean roots inoculated with Fusarium solani f.sp. glycines. Mol Genet Genomic 2002, 268:407-417.

72. Livak KJ, Schmittgen TD: Analysis of relative gene expression data using real-time quantitative PCR and the $2-\Delta \Delta C T$ method. Methods 2001 , 25:402-408

73. Zhao G, Wang J, Han Y, Teng W, Sun G, Li W: Identification of QTL underlying the resistance of soybean to pod borer, Leguminivora 
glycinivorella (Mats. Obraztsov), and correlations with plant, pod and seed traits. Euphytica 2008, 164:275-282.

74. Schmutz J, Cannon SB, Schlueter J, Ma J, Mitros T, Nelson W, Hyten DL, Song Q, Thelen JJ, Cheng J, Xu D, Hellsten U, May GD, Yu Y, Sakurai T,

Umezawa T, Bhattacharyya MK, Sandhu D, Valliyodan B, Lindquist E, Peto M, Grant D, Shu S, Goodstein D, Barry K, Futrell-Griggs M, Abernathy B, Du J, Tian Z, Zhu L, Gill N: Genome sequence of the palaeopolyploid soybean. Nature 2010, 463:178-183.

75. Zhang J, Yu O: Metabolic engineering of isoflavone biosynthesis in seeds. In Modification of seed composition to promote health and nutrition. In Agronomy Monograph Series. Edited by Krishnan H. ; 2009:151-177.

76. Lander ES, Green P, Abrahamson J, Barlow A, Daly M, Lincoln S, Newburg L: Mapmaker: an interactive computer package for constructing primary genetic linkage maps of experimental and natural populations. Genomics 1987, 1:174-181.

77. Zeng Z: Theoretical basis of separation of multiple linked gene effects on mapping quantitative trait loci. Proc Natl Acad Sci U S A 1993, 90:10972-10976.

78. Voorrips R: MapChart: software for the graphical presentation of linkage maps and QTL. J Hered 2002, 93:77.

79. Mehrabian M, Allayee H, Stockton J, Lum PY, Drake TA, Castellani LW, Suh M, Armour C, Edwards S, Lamb J, Lusis AJ, Schadt EE: Integrating genotypic and expression data in a segregating mouse population to identify 5lipoxygenase as a susceptibility gene for obesity and bone traits. Nat Genet 2005, 37:1224-1233.

80. Sonderby IE, Hansen BG, Bjarnholt N, Ticconi C, Halkier BA, Kliebenstein DJ: A systems biology approach identifies a R2R3 MYB gene subfamily with distinct and overlapping functions in regulation of aliphatic glucosinolates. PLoS One 2005, 2:e1322.

81. Moscou MJ, Lauter N, Steffenson B, Wise RP: Quantitative and qualitative stem rust resistance factors in barley are associated with transcriptional suppression of defense regulons. PLoS Genet 2011, 7:e1002208.

doi:10.1186/1471-2164-15-680

Cite this article as: Wang et al.: Expression quantitative trait loci infer the regulation of isoflavone accumulation in soybean (Glycine max $\mathrm{L}$. Merr.) seed. BMC Genomics 2014 15:680.

\section{Submit your next manuscript to BioMed Central and take full advantage of:}

- Convenient online submission

- Thorough peer review

- No space constraints or color figure charges

- Immediate publication on acceptance

- Inclusion in PubMed, CAS, Scopus and Google Scholar

- Research which is freely available for redistribution

Submit your manuscript at www.biomedcentral.com/submit
C Biomed Central 